\title{
ORIENTACIÓN AL \\ MERCADO E INNOVACIÓN \\ EN LAS EMPRESAS \\ DEL SECTOR SERVICIOS \\ EN LA CIUDAD \\ DE VILLAVICENCIO
}

RESUMEN

El proceso de orientación al mercado (om) permite generar valor para los clientes en la medida que se compensan las preferencias y las necesidades de la mejor manera en el mercado, posibilitando el desarrollo de las ventajas competitivas sostenibles y perdurables en el tiempo. La om tiene dos dimensiones que la tipifican: una de carácter cognitivo, que presenta una dicotomía entre la filosofía/cultura de negocio de un lado, y el conocimiento/inteligencia; del otro, la dimensión conductual se divide a su vez en una perspectiva basada en procesos y la otra, basada en la función/acción como misión de la OM. La OM requiere continuos esfuerzos de innovación a fin de permitir que la organización pueda adaptarse a las exigencias de su entorno. Esta innovación -llamada blanda-, desde los postulados del marketing internacional, está relacionada con procesos y es direccionada a la gestión organizacional y al desarrollo de procesos técnicos productivos.

Palabras clave: Orientación; Mercado; Innovación; Marketing; Internacional. 


\section{RAFAEL OSPINA INFANTE * \\ JENNY MILENA RIVEROS CASTAÑEDA *}

\section{MARKET ORIENTATION AND INNOVATION IN THE SERVICE SECTOR FIRMS IN THE CITY OF VILLAVICENCIO}

\section{ABSTRACT}

The process of market orientation (Mo) can generate value for customers to the extent that the preferences and needs of the best on the market are offset, enabling the development of sustainable and enduring competitive advantages over time. The mo has two dimensions that typify; cognitive nature of presenting a dichotomy between philosophy / culture business aside, and knowledge / intelligence, another behavioral dimension is subdivided into a process-based perspective and the other based on the function / action mission of mo.

The Mo require continuous innovation efforts allowing the organization to adapt to the demands of their environment. This innovation -called softwood-from the principles of international marketing, is related to processes and is directed to the organizational management to the development of technical production processes.

Keywords: Orientation; Market; Innovation; Marketing; International.

\section{INTRODUCCIÓN}

Es innegable la incidencia que ha tenido el proceso de globalización tanto en la definición de políticas públicas para la promoción de la actividad empresarial industrial en el municipio de Villavicencio, como en las decisiones empresariales de búsqueda de nuevos mercados y los procesos de innovación. Para ello ha sido necesario hacer exhaustivos análisis sobre los factores de competitividad y productividad con los cuales hacer frente a los retos que se le han impuesto a la economía nacional. En los pocos casos en los que esta dificultad estratégica ha sido superada, no ha sido posible traducir tal comprensión de los mercados en estrategias y programas de marketing que respondan de manera oportuna y eficiente a los requerimientos de los segmentos de mercados extranjeros en los que han incursionado. Las acciones emprendidas han obedecido a las clásicas y tradicionales decisiones que muchas empresas han tomado indistintamente del sector económico en el que han estado operando. En consecuencia, han sido pocas, por no decir casi nulas, las respuestas innovadoras implementadas. En el municipio de Villavicencio, departamento del 
Meta la situación no ha sido tan distinta de lo que ha sucedido en el país. No solo es escaso el número de empresas que han decidido tomar ventaja de los beneficios que se obtienen al incursionar en mercados foráneos, sino que las pocas que se han decidido a hacerlo no han alcanzado el éxito esperado. Ninguna de ellas ha logrado conocer las razones por las cuales sus estrategias de marketing no han funcionado en el dinámico y cambiante mercado en el que se han movido. Aún más difícil ha sido la realización de innovaciones de gestión en las estrategias de mercadeo. Se han dedicado a ensayar estrategias que por su estandarización -sin reflexión- no han logrado llegar de manera oportuna y eficiente a los mercados en los que han incursionado. Ante esta inobjetable realidad de las empresas del sector de servicios regionales surge la siguiente pregunta de investigación: ¿Cuál es la relación que existe entre la orientación al mercado e innovación en las empresas del sector servicios en la ciudad de Villavicencio, en el departamento del Meta?

\section{FUNDAMENTOTEÓRICO}

Como requisito ineludible para encontrar la relación existente entre orientación al mercado e innovación en las empresas del sector servicios en la ciudad de Villavicencio y los procesos de innovación que se han desarrollado en su interior, es necesario precisar los aspectos teóricos más relevantes. En primer lugar, debe definirse con exactitud el concepto de orientación al mercado, sus componentes y algunos debates epistemológicos desarrollados a su alrededor. En segundo lugar, debe delimitarse el concepto de innovación solo a los aspectos de gestión y de organización. Para terminar, también es relevante mostrar las principales características de las estrategias y los programas de marketing que sirven para entender el tema de la estandarización de las decisiones tomadas.

\section{LA ORIENTACIÓN AL MERCADO}

Al constructo de orientación al mercado se le ha dado una gran importancia recientemente. Como temática de estudio para la enseñanza y práctica del marketing, ha venido siendo reconocida desde hace varias décadas. Los académicos han venido dando gran significado a la importancia de incrementar el grado de orientación al mercado de las empresas como forma de obtener mejores resultados dentro de la organización. Diferentes estudios han demostrado que las empresas que han evidenciado un mayor grado de orientación al mercado son más rentables (Kohli y Jasworski, 1990, 1993b). Según Varela y Clavo (1998), la orientación al mercado se ha transformado en la piedra angular de las actuaciones de los responsables del marketing. Se ha evidenciado que las empresas orientadas al mercado tienen un mayor énfasis externo, los procesos existentes se realizan de afuera hacia dentro, y de manera especial se ha reiterado la necesidad de comprender los mercados y solidificar la relación con los consumidores y con los canales de distribución (Day, 1994). Desde esta perspectiva, la orientación al mercado supone la mezcla de dos aspectos organizacionales: funcional y estratégico. Ello significa que una empresa orientada al mercado es la que: 1) asume como filosofía de gestión integral el concepto de marketing, 2) traduce orgánicamente dicha filosofía en forma de cultura organizativa y 3 ) aplica operativamente este concepto (Martín, 2003 , p. 8). El proceso de orientación al mercado permite la generación de valor para los clientes, en la medida en la que se compensan las preferencias y las necesidades de la mejor manera en el mercado, con el objetivo de posibilitar el desarrollo de las ventajas competitivas sostenibles y perdurables en el tiempo. La orientación al mercado, para Felton (1959), es el estado de ánimo corporativo que subraya la integración y la organización de todas las funciones de marketing, las cuales se funden y se 
coordinan con el resto de funciones corporativas para alcanzar el fin último de la organización plasmado en el beneficio máximo posible. Para Kohli y Jasworski (1993), la orientación al mercado consiste en la generación de información del mercado respecto a las necesidades actuales y futuras de los clientes, la diseminación de dicha información a todos los departamentos, y el diseño e implantación de acciones de respuesta por parte de toda la empresa. Para Narver y Slater (1991, 1998), la conducta empresarial se plasma en tres componentes esenciales: orientación al cliente, orientación a la competencia y coordinación e integración de funciones (coordinación interfuncional). Estos pilares, a su vez, se hallan enmarcados en dos criterios de decisión gerencial: impacto en el largo plazo e impacto en la rentabilidad. Para Day (1994), la cultura debe centrarse en la captación de la información detallada del mercado y la necesidad de llevar a cabo acciones organizativas coordinadas y dirigidas al logro de ventajas competitivas sostenibles. Para Lambin (1995), la estrategia competitiva involucra a todas las fuerzas participantes del mercado (cliente final, cliente intermedio, competencia y entorno) y a todos los niveles. Por su parte, Cadogan y Diamontapulos (1995) plantean una reconceptualización del constructo de orientación al mercado, integrando los aportes de Naver y Slater y los de Kohli y Jaworsk. Sitúan como elemento central los mecanismos de organización intrafuncional (en el interior de la organización) e interfuncional (en relación con el mercado). Sus componentes son los determinados por Kohli y Jaworski con el énfasis de Naver y Slater en los aspectos relacionados con el cliente y la competencia. Algunos definen la dirección orientada al mercado como un proceso mediante el cual los aspectos relativos al mercado son incorporados al diseño de los diferentes sistemas de dirección. Añaden que la orientación al mercado se refiere al grado en el que los sistemas de dirección organizativa se encuentran diseñados para promover la orientación del negocio hacia sus clientes y hacia sus competidores. Tuominen, Rajala, y Möller (2004), basados en el concepto de aprendizaje organizacional, proponen dos dimensiones: una cognitiva y otra conductual. La dimensión cognitiva presenta una dicotomía que comprende, en un extremo, la filosofía/cultura de negocio, y en el otro, el conocimiento/inteligencia. La dimensión conductual se divide, a su vez, en una perspectiva basada en los procesos y la otra, circunscrita a la función/acción, como misión de la orientación al mercado.

\section{MODELOS BASADOS EN LAADOPCIÓN DE INNOVACIONES EN MERCADEO}

En relación con la innovación, se desarrollaron varios estudios en paralelo a los realizados por la escuela de Uppsala, centrados en la dinámica del proceso de desarrollo exportador, y apoyados en Rogers (1983), sobre todo en "la difusión de la innovación que relacionaba la expansión de la innovación con la interacción social". Según estos estudios, la decisión depende de la información de que dispone la empresa (Lee y Brash, 1978). Entrar a un mercado depende de la facilidad de conseguir información acerca del mercado a atender. En la medida en que las empresas de un país vayan entrando en nuevos mercados internacionales, el flujo de información del sector mejora. Y esta información es la que permite que otras empresas tomen la decisión de internacionalizarse, debido a que la acumulación de información reduce la incertidumbre. En el interior de las empresas, la dirección establece altos grados de prevención del riesgo incorporado al eventual fracaso de un producto. Este recelo se hace más evidente a la hora de introducir productos novedosos que traten de satisfacer las nuevas necesidades y los nuevos deseos de los consumidores. $Y$ es aún más incierto cuando se trata de modificar ciertos procesos que conduzcan a la organización 
a tener más elevados grados de orientación al mercado. En este sentido, el grado de orientación al mercado de una organización se encuentra enmarcado por un conjunto de factores identificados como antecedentes y determinantes, tales como aquellos relativos a la dinámica y al carácter de las relaciones existentes entre las diferentes áreas de la organización. La existencia de una relación coordinada y ausente de tensiones entre los distintos departamentos fomentará la orientación hacia el mercado de la organización (Kohki y Jaworski, 1993). Al respecto, algunos autores señalan la existencia de conflicto entre los departamentos como un inhibidor de la orientación al mercado (Levitt, 1969; Lush et al., 1976; Felton, 1959). Las tensiones generadas entre las distintas áreas de la organización, si bien no afectan al proceso de adquisición de información y generación de una inteligencia de marketing, sí limitan la comunicación inter-departamental y dificultan el proceso de diseminación de la inteligencia de marketing (Rueker y Walker, 1987). Jaworski y Kohli (1993) confirman este hecho, al sostener que un alto grado de conflicto entre los departamentos de una organización no solo afecta su correcto desarrollo, sino que retrasa el ofrecimiento de una respuesta adecuada por parte de la empresa. Se espera que un elevado grado de interconexión en la empresa facilitará el proceso de diseminación de la inteligencia de marketing $\mathrm{y}$, por ende, el ofrecimiento de respuesta que se corresponda con la realidad de cada mercado (Jaworski y Kohli, 1993). Las dinámicas características del mercado requieren la implementación de una nueva respuesta por parte de la empresa. Así, la existencia de una estructura altamente formalizada (las normas, roles y relaciones de autoridad) dificultará el proceso de innovación del comportamiento (ídem). Las estructuras empresariales potentemente centralizadas en la organización afectan la dinámica de generación y diseminación de inteligencia de marketing, además de la correcta consumación de una respuesta al mercado. Por ende, las organizaciones que se caracterizan por una dirección encomendada del proceso de toma de decisión, una elevada prevención al riesgo asociado a la innovación y un bajo grado de orientación al consumidor, arrojarán como resultado una baja orientación al mercado. La orientación al mercado representa un tipo concreto de cultura organizativa en la que el consumidor es centro de la estrategia y de las operaciones (Deshpandé y Webster, 1989). El alto poder de negociación de proveedores, distribuidores y consumidores, el grado de innovación tecnológica del mercado, la existencia de barreras de entrada al sector y la intensidad de la competencia, son algunos de los factores que ponen en peligro la supervivencia de la empresa en los mercados. Se trata, en definitiva, de un conjunto de creencias y convicciones acerca de cuál debe ser el funcionamiento de la organización (Martín, 2003, pág. 42). Para Slater y Narver (1995), la orientación al mercado es una cultura en la que se otorga la máxima prioridad a la creación y mantenimiento de valor óptimo para los consumidores, de modo rentable. Se parte de los intereses de los elementos e integrantes de la organización y, al tiempo y coordinadamente, en otra dirección, se proveen normas de gestión en relación con el desarrollo organizativo y la respuesta a las demandas del mercado.

\section{LA ORIENTACIÓN AL MARKETING}

La confusión que se ha generado en algunos círculos académicos y empresariales en relación con el constructo de orientación al mercado (nacional o internacional) y el de orientación al marketing, obliga a elaborar un marco conceptual preliminar que sirva de guía para el desarrollo de la investigación. Una orientación al marketing supone la aplicación de modelos estratégicos organizacionales cuyo eje central sea el clásico modelo del marketing mix, o de las 4 p's, que ha sido cuestionado en diferentes 
escenarios académicos ( Marion et al., 2003). Desde esta perspectiva, los resultados de las empresas dependen del control que se tenga sobre las variables del marketing. La diferenciación y adaptación de un producto a las necesidades del mercado se ha transformado en una decisión estratégica de significativo impacto en el posicionamiento de las empresas en los mercados foráneos. No obstante que ello demanda la utilización de la tecnología adecuada (Cavusgil et al., 1993), es la orientación a los mercados, desde el inicio del proceso de internacionalización, la que les ha permitido participar en los mercados con bienes innovadores ( Mc Duugall et al., 1994). Según Hymer, la posibilidad de obtener éxito en el mercado con un producto diferenciado con tecnología incorporada, calidad, prestigio de marca y cautela sobre el precio se logra mediante adecuados programas de marketing que sirvan para persuadir a los consumidores. Mora (2000) indica que las empresas españolas pueden tener distintas estrategias para ser competitivas: invertir recursos en programas de marketing, desarrollo y/o tecnología, y reducción de costos con la finalidad de ser competitivos en el ámbito internacional. La diferenciación es importante para obtener éxito en este proceso y esta se logra a través de las variables del mix de marketing.

\section{METODOLOGÍA}

\section{METODOLOGÍA DE LA INVESTIGACIÓN}

Esta investigación usará las diferentes referencias teóricas de las investigaciones más relevantes en los temas de innovación en mercadeo y orientación al mercado, con la finalidad de entender la diversidad y amplitud del tema en las organizaciones dedicadas a los procesos del sector servicios en el departamento del Meta. Esta investigación se hará bajo un enfoque cualitativo en su expresión de teoría fundada, que implica la búsqueda de modelos teóricos que den cuenta de la problemática experimentada por las empresas Ilaneras del sector servicios. La recopilación de la información primaria se hará recurriendo a las tres técnicas conocidas para este tipo de investigación: la observación, la entrevista en profundidad semiestructurada y las bases de datos disponibles en los archivos de las empresas y de las organizaciones e instituciones regionales relacionadas con empresas del sector servicios. La presente investigación tendrá un carácter comprensivo del fenómeno a estudiar y no explicativo de posibles relaciones de causa-efecto. Se apoyará en la inducción como método de análisis de las diferentes piezas de información recopiladas y no en la deducción hipotético-factorial que establezca variables dependientes o independientes y la incidencia de unas sobre otras. Esta definición metodológica se fundamenta en el hecho de no existir, hasta el momento, modelos y teorías que den cuenta de la actual situación de orientación al mercado que viven las empresas del sector servicios en el municipio de Villavicencio, capital del departamento del Meta. La finalidad de comprender estos procesos dentro de las empresas del sector servicios se recurrirá al análisis incremental de la información que se vaya recopilando, durante todo el tiempo -trabajo de campo, validación, análisis, categorización-: esta investigación seguirá fielmente la perspectiva estructurada propuesta por Miles y Huberman (2003). Es decir, en la medida en la que se va recopilando la información se va analizando e interpretando hasta llegar a la denominada saturación teórica (Glaser \& Strauss, 1967).

\section{RECOLECCIÓN DE INFORMACIÓN}

Las entrevistas semiestructuradas se realizarán en principio a los 25 gerentes generales, socios de empresa o gerentes de mercadeo en las empresas del sector servicios identificados en el municipio de Villavicencio, capital del depar- 
tamento del Meta, hasta llegar a la saturación teórica. Una vez realizada la primera entrevista se procesa la información, se codifican y analizan los datos, y después se redefine la estructura de la siguiente entrevista y así sucesivamente hasta obtener la información requerida que permita comprender el fenómeno investigado. La recolección de testimonios es controlada como en la teoría emergente (Glaser, 1978), es decir, a través del descubrimiento de las categorías de análisis que a su vez dan cuenta de la realidad vivida por las empresas estudiadas. Una vez realizado todo el trabajo de campo y se hayan procesado las entrevistas y se haya obtenido la información requerida, se realiza un análisis histórico con datos oficiales de ventas y utilidades de las respectivas empresas, con la finalidad de obtener indicadores de la gestión de los últimos años, lo cual permitirá completar y comprender el análisis de todo el fenómeno en estudio. Se desarrollarán entrevistas en profundidad, individuales y semiestructuradas; se hará observación participante y no participante en el momento de tomar las decisiones respectivas, y se analizarán los datos históricos acumulados respecto a las dos actividades principales de estudio: la orientación al mercado y la innovación. Todo esto será registrado en audio, en video, transcrito y analizado, para establecer las categorías correspondientes.

\section{ANÁLISIS DE DATOS}

En esta investigación se realizará el análisis, se comprenderán los datos y fenómenos, recurriendo a diferentes técnicas de recolección de información establecidas por la teoría fundada (Glaser \& Strauss, 1967). De la misma manera, toda la información recopilada será confrontada y validada con actores del proceso, a fin de reducir los sesgos propios de la misma re- copilación de los datos. Para ello se realizará un proceso incremental que se desarrollará a manera de espiral entre los datos encontrados y la corroboración y validación de las teorías que fundamentan la innovación en mercadeo y la orientación al mercado. Todo el cúmulo de información recopilada será sometido a rigurosos análisis propios de la teoría fundada. Se transcribirán de manera literal los datos, se les hará un análisis microscópico (línea por línea), se les aplicará un análisis axial, se establecerán las categorías, se extractarán los constructos y se diseñarán los modelos que vayan emergiendo en el contraste entre la teoría y la realidad social estudiada. La información a analizar tendrá un carácter convergente, acumulativo. Es decir, el proceso de captación y de comprensión de la realidad exige que todas las piezas de información se pongan juntas a fin de ir descubriendo las interrelaciones teóricas y conceptuales que surjan. Se recurrirá también a los diarios de campo en los que los investigadores registren sus apreciaciones después de cada ejercicio de recopilación de datos, siendo piezas fundamentales en el momento de analizar el comportamiento de lo estudiado en su escenario natural de comportamiento.

\section{RESULTADOS}

Como resultado del proceso investigativo mediante el estudio de enfoque de teoría fundada para comprender el fenómeno de Orientación al mercado e innovación en las empresas del sector servicios en la ciudad de Villavicencio, llevado a cabo, a continuación se hace una reflexión sobre la orientación al mercado y la innovación en mercadeo internacional, lo que ha involucrado y representa para las empresas del sector servicios y lo que seguirá simbolizando en estas organizaciones. 


\section{ORIENTACIÓN AL MERCADO}

Las empresas del sector servicios en la ciudad de Villavicencio, a pesar de lo simplificado que pueda ser su estructura organizacional, se encuentran orientadas al mercado; la razón es que estas organizaciones han logrado apropiar la filosofía de marketing en forma de cultura organizacional en la cual se han establecido, fortalecido y desarrollado relaciones de largo plazo con sus clientes, proveedores y coordinación, logrando actitudes y comportamientos para la creación de valor en la organización interna, desarrollando una cultura en pro de la om que proporciona de manera general resultados positivos generales y aumento de ventas; esto, además de las bondades ya mencionadas, les permite a las empresas del sector servicios en la ciudad de Villavicencio enfrentar la turbulencia de macroentorno que cada vez es más dinámica.

\section{ORIENTACIÓN AL CLIENTE}

En oposición a lo que tradicionalmente se cree que una empresa necesita usar técnicas de investigación de mercados para conocer las necesidades de los clientes, las empresas del sector servicios en la ciudad de Villavicencio conocen no solo las necesidades actuales de sus clientes si no las potenciales. Esto lo han logrado gracias a las relaciones cercanas de largo plazo o marketing relacional que han logrado con ellos, lo que ha permitido construir valor para el cliente, satisfacción y, por ende, lealtad con la organización, mediante la estrategia de combinación de atención al cliente personalizada, precio y calidad.

\section{ORIENTACIÓN A LA COMPETENCIA}

Las empresas del sector servicios en la ciudad de Villavicencio, contrario de lo que se cree, producto de las múltiples barreras que enfrenta en mercados internacionales, conocen perfectamente quién es su competencia local, nacional e internacional, además de comprender su nivel competitivo, lo cual les admite analizar y comprender sus acciones y estrategias, permitiendo reacciones frente a las estrategias de la competencia con una combinación estratégica que involucra planeación, innovación, calidad y servicio personalizado, para generar una percepción superior de valor entre sus clientes.

\section{RELACIÓN INTERFUNCIONAL}

Las empresas del sector servicios en la ciudad de Villavicencio han logrado coordinar inter-

Proceso de Innovación en las empresas del sector servicios en la ciudad de Villavicencio

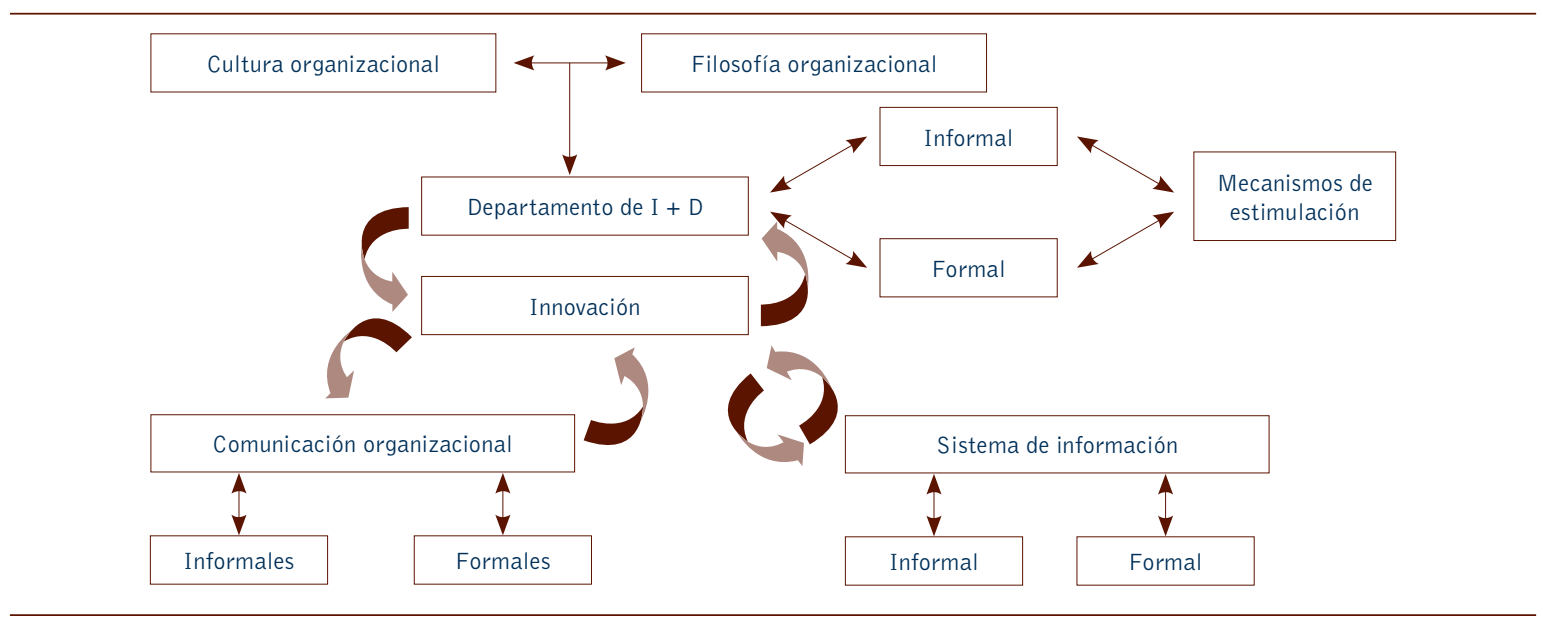

Fuente: Elaboración propia. 
funcionalmente el desarrollo de la cultura organizacional, con el objetivo de generar valor superior para el cliente, logrando una ventaja competitiva. Esto se ha logrado a pesar de no contar con una estructura organizacional y sistema de información formal, pero la acumulación de experiencia les ha permitido analizar sus experiencias y comportamientos del mercado y comunicarlo de manera eficiente.

En la innovación y la orientación al mercado de las empresas del sector servicios en la ciudad de Villavicencio, existen relaciones cercanas de largo plazo en el desarrollo de sus actividades empresariales: Ias RCLP sociales establecen vínculos con clientes internos y externos y la competencia, en espacios sociales, con la finalidad de identificar y conocer fortalezas, características y roles, estableciendo una especie de "ropaje" e instituyendo relaciones de confianza y beneficio mutuo, definiendo territorios y fronteras de los mismos (RCLP espaciales), para alcanzar la maximización de valor de la empresas. Estas relaciones tienen como fin último la rentabilidad económica para las empresas (RCLP económica). Las relaciones cercanas de largo plazo no son exclusivas del departamento de marketing: son transversales a la organización y parte fundamental de la filosofía y cultura organizacional de las empresas del sector servicios en la ciudad de Villavicencio.

\section{RELACIONES CERCANAS DE LARGO PLAZO}

Se evidenciaron en las empresas del sector servicios en la ciudad de Villavicencio las relaciones cercanas de largo plazo (RCLP), como un eje común entre los fenómenos estudiados acerca de la orientación al mercado e innovación en mercadeo de estas organizaciones, lo que permite la generación de valor en el desarrollo de las actividades de mercadeo e innovación, lo cual es resultado de interacciones sociales entre clientes, competencia y funcionarios, generando un sistema informal y oral que permite captar información de insumo fundamental para el diseño de estrategias de mercadeo y mejoras continuas de productos y procesos. Las relaciones cercanas de largo plazo que establecen con las empresas del sector servicios en la ciudad de Villavicencio, la innovación y la orientación al mercado contienen tres dimensiones: sociales, espaciales y económicas.

Categorías en innovación en mercadeo de las empresas del sector servicios en la ciudad de Villavicencio

\begin{tabular}{l|c|c|c}
\hline \multicolumn{1}{c|}{ Tipología } & Científico & Participativo & Aprendiz \\
\hline $\begin{array}{l}\text { Estructura } \\
\text { organizacional }\end{array}$ & $\begin{array}{c}\text { Formal y } \\
\text { estructurada }\end{array}$ & $\begin{array}{c}\text { Planay } \\
\text { simplificada solo } \\
\text { departamento } \\
\text { de producción }\end{array}$ & $\begin{array}{c}\text { Plana y } \\
\text { simplificada }\end{array}$ \\
\hline $\begin{array}{l}\text { Departamento } \\
\text { de innovación } \\
\text { y desarrollo }\end{array}$ & Sí & $\begin{array}{c}\text { Só- } \\
\text { Coinvestigación }\end{array}$ & No \\
\hline $\begin{array}{l}\text { Procesos de } \\
\text { innovación } \\
\text { incremental }\end{array}$ & Sí & Sí & No \\
$\begin{array}{l}\text { Innovación en } \\
\text { procesos }\end{array}$ & Sí & Sí & Sí \\
\hline $\begin{array}{l}\text { Sistemas de } \\
\text { calidad }\end{array}$ & Sí & Sí & No \\
\hline $\begin{array}{l}\text { Sistema de } \\
\text { información } \\
\text { en mercadeo }\end{array}$ & Formal & Informal & Informal \\
\hline
\end{tabular}

Fuente: Los autores.

Relación entre la innovación en mercadeo y la orientación al mercado

\begin{tabular}{|c|c|c|}
\hline $\begin{array}{l}\text { Tipología de orientación } \\
\text { al mercado }\end{array}$ & & $\begin{array}{c}\text { Tipología } \\
\text { de innovación } \\
\text { en mercado }\end{array}$ \\
\hline $\begin{array}{l}\text { Estructuración Relacionista } \\
\text { Sobreviviente Filósofo Formador }\end{array}$ & $\begin{array}{c}\text { Relaciones cercanas } \\
\text { de largo plazo: } \\
\text { Económias Sociales } \\
\text { Espaciales }\end{array}$ & $\begin{array}{c}\text { Científico } \\
\text { Participativo } \\
\text { Aprendiz }\end{array}$ \\
\hline
\end{tabular}

Fuente: La autora 


\section{PRINCIPALES CONTRIBUCIONES}

Muy a pesar de que las empresas del sector servicios en la ciudad de Villavicencio no cuentan con un departamento de investigación y desarrollo, tecnología de punta y sistema de información formal, se han evidenciado en todas estas organizaciones procesos de innovación blandas $y / 0$ incrementales producto de una cultura innovadora relacionada estrechamente con el aprendizaje organizacional, derivado de analizar el mercado, comportamiento y experiencias del talento humano que las componen.

En la innovación y la orientación al mercado de las empresas del sector servicios en la ciudad de Villavicencio existen relaciones cercanas de largo plazo: en el desarrollo de sus actividades empresariales las RCLP sociales establecen vínculos con clientes internos y externos y la competencia, en espacios sociales, con la finalidad de identificar y conocer fortalezas, características y roles, estableciendo una especie de "ropaje" e instituyendo relaciones de confianza y beneficio mutuo, definiendo territorios y fronteras de los mismos (RCLP ESpaciales), en búsqueda de la maximización del valor de la empresas. Estas relaciones tienen como fin último la rentabilidad económica para las empresas (RCLP económica). Las relaciones cercanas de largo plazo no son exclusivas del departamento de marketing: son transversales a la organización y parte fundamental de su filosofía y cultura organizacional.

\section{RELACIONES CERCANAS DE LARGO PLAZO}

Se evidenciaron en las empresas del sector servicios en la ciudad de Villavicencio las relaciones cercanas de largo plazo (RCLP) como un eje común entre los fenómenos estudiados acerca de la orientación al mercado e innovación en mercadeo de estas organizaciones, lo que permite la generación de valor en el desarrollo de las actividades de mercadeo e innovación. Esto es el resultado de interacciones sociales entre clientes, competencia y funcionarios, generando un sistema informal y oral que permite captar información de insumo fundamental para el diseño de estrategias de mercadeo y mejoras continuas de productos y procesos. Las relaciones cercanas de largo plazo que establecen con las empresas del sector servicios en la ciudad de Villavicencio, la innovación y la orientación al mercado contienen tres dimensiones: sociales, espaciales y económicas.

\section{CONCLUSIONESY DISCUSIÓN}

Para una completa comprensión e interpretación de los hallazgos de los objetivos desarrollados en la investigación, es inevitable hacer una reflexión final de algunas limitaciones que enfrentó esta investigación en su desarrollo, además de conocer los alcances y algunos futuros fenómenos de investigaciones que pueden surgir producto de esta.

\section{ALGUNAS LIMITACIONES}

La ejecución de la investigación orientación al mercado e innovación de las empresas del sector servicios en la ciudad de Villavicencio, en el departamento del Meta, no está exenta de limitaciones: sin que estas lograran impedir su construcción y conclusiones generales, sí se generaron algunos conflictos para su abordaje.

En primer lugar, el reducido número de las empresas del sector servicios en la ciudad de Villavicencio, en el departamento del Meta, 5 en total, su pequeño tamaño y simplificadas estructuras organizacionales dificultó la recolección de información de varias fuentes en una misma empresa; aunque se realizó la recolección de información, hizo más complejo el análisis de la misma. 
En segundo lugar, la experiencia del proceso de prestación del servicio y el número de operaciones realizadas por empresas son relativamente pocas, así como los destinos de las mismas. Fueron realizadas las entrevistas y tomado el material fotográfico y audiovisual y se encontró información suficiente y rica para cumplir el objetivo del estudio.

En tercer lugar, el no haber obtenido información primaria de los proveedores de los competidores, así como del canal y sus clientes, permitió la comprensión del fenómeno desde la óptica de la organización, lo cual no imposibilitó la realización de un análisis profundo, riguroso, comprehensivo, del fenómeno estudiado.

\section{ALCANCES}

Los objetivos que han llevado a la comprensión del fenómeno de orientación al mercado e innovación de la zona de estudio, debe ser extensivo solo de manera categórica, mas no estadística. Es decir, de ninguna manera estos resultados pueden considerarse como representativos de la realidad de otras ciudades, aunque estas sean similares en determinados aspectos. Debe entenderse que las investigaciones cualitativas con enfoque de teoría fundada son de carácter comprensivo y, en consecuencia, los resultados e interpretaciones no pueden distanciarse del contexto de donde fue tomada la información.

\section{FUTURAS INVESTIGACIONES}

La gran complejidad del estudio de la orientación al mercado e innovación en empresas exportadoras, que se hizo desde la rigurosidad académica, así como los hallazgos aquí diseñados conviene entenderlos apenas como un punto de partida para el estudio de este fenómeno. Por esta razón, las iniciativas encaminadas a ahondar en este tema en otros sectores o ciudades le corresponderá la realización de esfuerzos adicionales de investigación, con la misma metodología, si se quiere profundizar en los aspectos concluyentes aquí analizados.

\section{REFERENCIAS}

Aaker, D. (1988). Strategic Marketing Management. New York: John Wilwy and Sons, 2. ${ }^{a}$ ed.

Agarwal, S. \& Ramaswani, S.N. (1992). "Choice of Foreing Market Entry Mode: Impact of Ownership, Location and Internalization Factors", in Journal of International Business Studies, vol. 27, First Quarter, pp. 1-27.

Alonso, J. A. (1993). "Capacidades exportadoras y estrategia internacional de la empresa", en Velarde, J.; García, J.L.y Pedreño, A. Empresas y empresarios españoles en la encrucijada de los noventa. Ed. Civitas, pp. 205-258.

Álvarez, J. (2010). Innovación Desafío para el Desarrollo en el siglo XXI. Bogotá: Cátedra José Celestino Mutis, Universidad Nacional de Colombia.

Baker, T. L.; Simpson, P. M. \& Siguaw, J. A. (1999). "The Impact of Supplier. Perceptions of Reseller Market Orientation in Key Relationship Constructs", in Journal of Academy of Marketing Science, vol. 27 (1), pp. 50-57.

Balabanis, G.; Stables, R.E. \& Phillips, H.C. (1997). "Market Orientation in the Top 200 Britain Charity Organizations and its Impact on their Performance", in European Journal of Marketing, vol. 31, n. ${ }^{\circ}$ 8, pp. 583-603.

Brignall, Stan \& Ballantine, Joan (1996). "Performances measurement in services business revisited", in International Journal of Service Industry Management, vol. 7, n. ${ }^{\circ}$ 1. EE.uU.

Buckler, Sheldon \& Zien, Karen (1996). "The spirituality of innovation: learning from stories", in Journal of Product Innovation Management, vol. 13. EE.UU. 
Cadogan, J.W. \& Diamantopoulus, A. (1995). "Narver and Slater, Kohli and Jaworski and the market orientation construct: integration and internalization", in Journal of Strategic Marketing, vol. 3, n. ${ }^{\circ}$ 1. EE.UU.

Cooper, Robert \& Edgett, Scott (1996). "Critical success factors for new financial services", in Marketing Management. Fall. EE.uu.

Dalgic, T. (1998). "Dissemination of Market Orientation in Europe. A Conceptual and Historical Evaluation", in International Marketing Review, vol. 15 (1), pp. 45-60.

Davidson, W.H. (1980). "The location of foreing direct investment activity: Country caracteristics and Experience Effects", in Journal of International Business Studies, vol. 11, pp. 9-22.

Dawson, L.M. (1987). "Transfering Industrial Technology to Less Developed Countries", in Industrial Marketing Management, vol. 16, n. ${ }^{\circ}$ 4, november, pp. 265-271.

Day, G. (2000). "Managing Market Relationship", in Journal of the Academy of Marketing Science, vol. 28, n. ${ }^{\circ}$ 1, pp. 24-30.

Deng, S. \& Dart, J. (1999). "The Market Orientation of Chinese Enterprises During a Time of Transition", in European Journal of Marketing, vol. 33, n. ${ }^{\circ}$ 5/6, pp. 631-654.

Dinacyt (2003). Encuesta de actividades de innovación. Año de referencia 2003. Uruguay: Ministerio de Educación y Cultura, Dirección Nacional de Ciencia, Tecnología e Innovación.

Diamantopoulos, A. (1988). "Identifying Differences Between High and Low Involvement Exporters", in International Marketing Review, vol. 21, n. ${ }^{\circ}$ 5, october, pp. 18-23.

Diamantopoulos, A. \& Cadogan, J. (1996). "Internationalizing the market orientation construct: an in-depth interview approach", in Journal of Atrategic Marketing, n. ${ }^{\circ}$ 4, pp. 23-52.

Diamantopoulos, A. \& Hart, S. (1993). "Linking Market Orientation and Company Performance: Preliminary Evidence on Kohli ad Jaworski's
Framework", in Journal of Strategic Marketing, n. ${ }^{\circ} 1$, pp. 93-121.

Diamantopoulos, A. \& Schlegelmilch, B. (1994). "Linking Export Manpower to Export Performance: A Canonical Regression Analysis of European and U.S." Data in Cavusgil, S.T.y Axinn, C. (eds.). Advances in International Marketing. New York: Jai.

Drucker, Peter (1985). "La disciplina de la innovación", en Harvard Business Review, vol. 82, n. ${ }^{\circ} 8$ (agosto 2004), reimpr. Chile.

Fitzgerald, L.; Johnston, R.; Brignall, S.; Silvestro, R. \& Voss, C. (1991). Performance measurement in service business. Inglaterra: CIMA.

Golden, P.A.; Johnson, D.M. \& Smith, J.R. (1995). "Strategic orientation and marketing strategies in transition economies: a study of Russian firms", in Journal of Strategic Marketing, vol. 3, n. ${ }^{\circ}$ 1. EE.UU.

Grenley, G.E. (1995). "Forms of Market Orientation in U.K. Companies", in Journal of Marketing Studies, vol. 32, n. ${ }^{\circ}$ 1. England.

Hamel, Gary \& Getz, Gary (2004). "Cómo innovar en una era de austeridad", in Harvard Business Review, vol. 82, n. ${ }^{\circ} 8$ (agosto 2004). Chile

Kohli, A.K. \& Jaworski, B.J. (1993). "Market Orientation: Antecedents and Consequences", in Journal of Marketing, vol. 57, julio, pp. 53-70.

Narver, J.C. \& Slater, S.F. (1991). "Becoming More Market Oriented: An Exploratory Study of the Programmatic and Market-Back Approaches Marketing Science Institute", in Working Papers n. ${ }^{\circ}$ 91-128.

Narver, J.C. \& Slater, S.F. (1998). "Additional Thoughts on the Measurement of Market Orientation: A Comment in Deshpandé and Farley", in Journal of Market Focused Management, vol. 2, pp. 233-236.

Reinoso, J. (2009). Los Indicadores de Gestión y su relación con la Cultura Organizacional. Ibagué: Universidad del Tolima, Grupo de Investigación en Estrategia, Estructura y Cultura Organizacional ECO. 
Rodríguez, J. (2006). La Dinámica de la Innovación Tecnológica Modelo Hiper 666. Bogotá: Universidad Nacional de Colombia Sede Bogotá, Grupo de Investigación Complexus.

Varela González, J.A. y Clavo Silvosa, A.R. (1998). "Comportamiento orientado por el mercado: internacionalización y otros antecedentes seleccio- nados una investigación empírica", en Información comercial Española, n. ${ }^{\circ} 774$, noviembre pp. 37-52. Zapata, A. (2008). Gestión de la Cultura Organizacional. Bases Conceptuales para su implementación. Cali: Universidad del Valle, Facultad de Ciencias Económicas. 
